\title{
P Colonies and kernel P Systems
}

\author{
Erzsébet Csuhaj-Varjú · Marian \\ Gheorghe • Raluca Lefticaru
}

Received: date / Accepted: date

\begin{abstract}
P}$ colonies, tissue-like $\mathrm{P}$ systems with very simple components, have received constant attention from the membrane computing community and in the last years several new variants of the model have been considered. Another $\mathrm{P}$ system model, namely kernel $\mathrm{P}$ system, integrating the most successfully used features of membrane systems, has recently attracted interest and some important developments have been reported. In this paper we study connections among several classes of $\mathrm{P}$ colonies and kernel $\mathrm{P}$ systems, by showing how the behaviour of these $\mathrm{P}$ colony systems can be represented as kernel $\mathrm{P}$ systems. An example illustrates the way it is modelled by using $\mathrm{P}$ colonies and kernel $\mathrm{P}$ systems and some properties of it are formally proved in the later approach.
\end{abstract}

\section{Introduction}

Membrane systems were introduced in [22] as a new natural computing paradigm inspired by the compartmentalised structure of the living cells and the main bio-chemical interactions occurring within and across compartments. A monograph [23] presenting the main developments at the level of early 2000 was

E. Csuhaj-Varjú

Department of Algorithms and Their Applications, Faculty of Informatics, ELTE Eötvös Loránd University, Budapest 1117, Hungary

E-mail: csuhaj@inf.elte.hu

M. Gheorghe

School of Electrical Engineering and Computer Science, University of Bradford, Bradford BD7 1DP, United Kingdom

E-mail: m.gheorghe@bradford.ac.uk

R. Lefticaru

School of Electrical Engineering and Computer Science, University of Bradford, Bradford BD7 1DP, United Kingdom

E-mail: r.lefticaru@bradford.ac.uk 
published. A comprehensive description of the key research areas covering both theoretical aspects as well as applications appears in this handbook [26]. More specific developments may be found in research papers, referring to generalised forms of rewriting $\mathrm{P}$ systems [20], rewriting tissue P systems [21], control languages used for $\mathrm{P}$ systems [18].

A $P$ colony represents a membrane system model with communities of cells communicating with a shared environment by means of simple rules and using a limited number of symbols in each cell $[14,5]$. An overview of the main developments of the model is presented in [15], a recent survey of the area can be found in [3]. Two more $\mathrm{P}$ colony models are of interest in the context of this paper, namely $P$ colonies with senders and consumers, and $P$ colonies with evolving environment. The first model [4], considers specific communication rules acting in one single direction, either from the cell to the environment (sending cell) or vice-versa (consuming cell). The later model considers the case of an environment whereby its contents might be affected not only by the exchanges with cells, but also by some evolving rules acting similarly to rules of context-free Lindenmayer systems (L systems). The behaviour and the computational power of this model are investigated in [2].

Kernel $P$ systems ( $k P$ systems, for short) $[8,9]$ aim to integrate in a coherent and elegant manner some of the most successfully used features of $\mathrm{P}$ systems.

The $\mathrm{kP}$ system model is also supported by a modelling language, called $\mathrm{kP}$ Lingua, capable of mapping a $\mathrm{kP}$ system model specification into a machine readable representation. Furthermore, $\mathrm{kP}$ systems are supported by a software framework, kPWORKBENCH [13], which integrates a set of related simulation and verification methods and tools.

After being introduced $[8,9], \mathrm{kP}$ systems have been investigated from various research angles. Their relationships with other classes of membrane systems have been investigated - firstly, membrane systems with active membranes and neural-like membrane systems have been mapped into $\mathrm{kP}$ systems $[9,10]$, then generalised communicating $\mathrm{P}$ systems have been connected with $\mathrm{kP}$ systems [16]. Tools, such as kPWORKBEnch [13], have linked modelling aspects with formal verification and model checking $[6,13,7]$. Various applications have been considered, such as 3-colouring problem [11], sorting algorithms [9,7], simple broadcasting [13], or synthetic biology paradigms - genetic logic gates [12].

In this paper we continue the investigation of the $\mathrm{P}$ colony and $\mathrm{kP}$ system models by considering the relationship between them. We show how the behaviour of $\mathrm{P}$ colonies of arbitrary capacity, $\mathrm{P}$ colonies with senders and consumers, and $\mathrm{P}$ colonies with capacity 2 and evolving environment is mapped into corresponding equivalent $\mathrm{kP}$ systems. Finally, we consider the problem of modelling the synchronisation aspects occurring in a producer/consumer problem by using adequate $\mathrm{P}$ colony and $\mathrm{kP}$ systems, exhibiting equivalent behaviour. Formal properties are investigated using the model checking facilities provided by the $\mathrm{kPWORKBENCH}$ tool associated with $\mathrm{kP}$ systems. 
The paper is organised as follows. In Section 2 we introduce the definitions of a kernel $\mathrm{P}$ system and a $\mathrm{P}$ colony. In Section 3 we study the relationships among the above mentioned classes of $\mathrm{P}$ colonies and $\mathrm{kP}$ systems. Section 4 introduces the producer/consumer problem and investigates its representation using $\mathrm{P}$ colony with senders and consumers and $\mathrm{kP}$ systems, as well as some formal properties. Finally, Section 5 concludes the paper.

\section{Kernel P Systems and P Colonies - Main Concepts and Definitions}

Standard P system concepts and standard notions such as strings, multisets, rewriting rules, and computation are well-known and we refer to [23] for their formal notations and precise definitions. The $\mathrm{kP}$ system concepts and definitions used in this paper are from $[8,9]$. Some of them are slightly changed and this will be mentioned when these concepts are discussed.

\section{$2.1 \mathrm{kP}$ System Basic Definitions}

We start by introducing the concept of a compartment type utilised later in defining the compartments of a $\mathrm{kP}$ system.

Definition $1 T$ is a set of compartment types, $T=\left\{t_{1}, \ldots, t_{s}\right\}$, where $t_{i}=$ $\left(R_{i}, \sigma_{i}\right), 1 \leq i \leq s$, consists of a set of rules, $R_{i}$, and an execution strategy, $\sigma_{i}$, defined over $\operatorname{Lab}\left(R_{i}\right)$, the labels of the rules of $R_{i}$.

The compartments that appear in the definition of the $\mathrm{kP}$ systems will be constructed using compartment types introduced by Definition 1. Each compartment, $C$, will be defined by a tuple $(t, w)$, where $t \in T$ is the type of the compartment and $w$ the initial multiset of it. The types of rules and the execution strategies occurring in the compartment types will be introduced and discussed later.

Definition 2 A $k P$ system of degree $n$ is a tuple

$$
k \Pi=\left(A, \mu, C_{1}, \ldots, C_{n}, i_{0}\right)
$$

where $A$ is a finite set of elements called objects; $\mu$ defines the initial membrane structure, which is a graph, $(V, E)$, where $V$ are vertices indicating compartments of the $\mathrm{kP}$ system, and $E$ edges; $C_{i}=\left(t_{i}, w_{i}\right), 1 \leq i \leq n$, is a compartment of the $\mathrm{kP}$ system, as presented above; $i_{o}$ is the label of the output compartment, where the result is obtained. 


\section{$2.2 \mathrm{kP}$ System Rules}

Each rule occurring in a $\mathrm{kP}$ system definition has the form $r\{g\}$, where $r$ stands for the rule itself and $g$ is its guard. The guards are constructed using multisets over $A$, as operands, and relational or Boolean operators. The definition of the guards is now introduced. We start with some notations.

For a multiset $w$ over $A$ and an element $a \in A$, we denote by $|w|_{a}$ the number of objects $a$ occurring in $w$. Let us denote $\operatorname{Rel}=\{<, \leq,=, \neq, \geq,>\}$, the set of relational operators, $\gamma \in \operatorname{Rel}$, a relational operator, and $a^{n}$ a multiset. We first introduce an abstract relational expression.

Definition 3 If $g$ is the abstract relational expression denoting $\gamma a^{n}$ and $w$ a multiset, then the guard $g$ applied to $w$ denotes the relational expression $|w|_{a} \gamma n$.

The abstract relational expression $g$ is true for the multiset $w$, if $|w|_{a} \gamma n$ is true.

We consider now the following Boolean operators $\neg$ (negation), $\wedge$ (conjunction) and $\vee$ (disjunction). An abstract Boolean expression is defined by one of the following conditions

- any abstract relational expression is an abstract Boolean expression;

- if $g$ and $h$ are abstract Boolean expressions then $\neg g, g \wedge h$ and $g \vee h$ are abstract Boolean expressions.

The concept of a guard, introduced here, is a generalisation of the promotor and inhibitor concepts utilised by some variants of $\mathrm{P}$ systems.

Definition 4 If $g$ is an abstract Boolean expression containing $g_{i}, 1 \leq i \leq q$, abstract relational expressions and $w$ a multiset, then $g$ applied to $w$ means the Boolean expression obtained from $g$ by applying $g_{i}$ to $w$ for any $i, 1 \leq i \leq q$.

As in the case of an abstract relational expression, the guard $g$ is true with respect to the multiset $w$, if the abstract Boolean expression $g$ applied to $w$ is true.

Example 1 If $g$ is the guard defined by the abstract Boolean expression $\geq$ $a^{5} \wedge\left\langle b^{3} \vee \neg>c\right.$ and $w$ a multiset, then $g$ applied to $w$ is true if it has at least $5 a^{\prime}$ s and no more than $2 b^{\prime}$ s or no more than one $c$.

Definition $5 \mathrm{~A}$ rule from a compartment $C_{l_{i}}=\left(t_{l_{i}}, w_{l_{i}}\right)$ can have one of the following types:

- (a) rewriting and communication rule: $x \rightarrow y\{g\}$, where $x \in A^{+}$and $y$ has the form $y=\left(a_{1}, t_{1}\right) \ldots\left(a_{h}, t_{h}\right), h \geq 0, a_{j} \in A$ and $t_{j}, 1 \leq j \leq h$, indicates a compartment type from $T$ (see Definition 2) of compartments linked to the current one; $t_{j}$ might also indicate the type of the current compartment, $C_{t_{t_{i}}}$; if a link does not exist (i.e., there is no link between the two compartments in $E$ ) then the rule is not applied; if a target, $t_{j}$, refers to a compartment type that appears in more than one compartments connected to $C_{l_{i}}$, then one of them will be non-deterministically chosen; 
- (b) structure changing rules; the following types of rules are considered:

- (b1) membrane division rule: $[x]_{t_{l_{i}}} \rightarrow\left[y_{1}\right]_{t_{i_{1}}} \ldots\left[y_{p}\right]_{t_{i_{p}}}\{g\}$, where $x \in$ $A^{+}$and $y_{j} \in A^{*}, 1 \leq j \leq p$; the compartment $C_{l_{i}}$ will be replaced by $p$ compartments; the $j$-th compartment, $1 \leq j \leq p$, of type $t_{i_{j}}$ contains the same objects as $C_{l_{i}}$, but $x$, which will be replaced by $y_{j}$; all the links of $C_{l_{i}}$ are inherited by each of the newly created compartments;

- (b2) membrane dissolution rule: []$_{t_{l_{i}}} \rightarrow \lambda\{g\}$; the compartment $C_{l_{i}}$ will be destroyed together with its links;

- (b3) link creation rule: $[x]_{t_{l_{i}}} ;[]_{t_{l_{j}}} \rightarrow[y]_{t_{l_{i}}}-[]_{t_{l_{j}}}\{g\}$; the current compartment is linked to a compartment of type $t_{l_{j}}$ and $x$ is transformed into $y$; if more than one compartment of type $t_{l_{j}}$ exist and they are not linked with $C_{t_{l_{i}}}$, then one of them will be non-deterministically picked up; $g$ is a guard that refers to the compartment of type $t_{l_{i}}$;

- (b4) link destruction rule: $[x]_{t_{l_{i}}}-[]_{t_{l_{j}}} \rightarrow[y]_{t_{l_{i}}} ;[]_{t_{l_{j}}}\{g\}$; is the opposite of link creation and means that the compartments are disconnected.

When in a rewriting and communication rule one of the right hand side elements $\left(a_{j}, t_{j}\right), 1 \leq j \leq h$, is such that $t_{j}=t_{l_{i}}$ then this is simply written as $a_{j}$.

The membrane division is defined slightly differently here compared to $[8$, $9]$, where each $y_{j}, 1 \leq j \leq p$, is composed of objects with target compartments.

In this paper we will be using only rewriting and communication rules (a).

\section{$2.3 \mathrm{kP}$ System Execution Strategies}

In $\mathrm{kP}$ systems the way in which rules are executed is defined for each compartment type $t$ from $T$ - see Definition 1. As in Definition 1, $L a b(R)$ is the set of labels of the rules $R$.

Definition 6 For a compartment type $t=(R, \sigma)$ from $T$ and $r \in \operatorname{Lab}(R)$, $r_{1}, \ldots, r_{s} \in L a b(R)$, the execution strategy, $\sigma$, is defined by the following

$-\sigma=\lambda$, means no rule from the current compartment will be executed;

$-\sigma=\{r\}-$ the rule $r$ is executed;

$-\sigma=\left\{r_{1}, \ldots, r_{s}\right\}$ - one of the rules labelled $r_{1}, \ldots, r_{s}$ will be non-deterministically chosen and executed; if none is applicable then nothing is executed; this is called alternative or choice;

$-\sigma=\left\{r_{1}, \ldots, r_{s}\right\}^{*}$ - the rules are applied an arbitrary number of times (arbitrary parallelism);

$-\sigma=\left\{r_{1}, \ldots, r_{s}\right\}^{\top}$ - the rules are executed according to the maximal parallelism strategy;

$-\sigma=\sigma_{1} \& \ldots \& \sigma_{s}$, means executing sequentially $\sigma_{1}, \ldots, \sigma_{s}$, where $\sigma_{i}, 1 \leq$ $i \leq s$, describes any of the above cases; if one of $\sigma_{i}$ fails to be executed then the rest is no longer executed;

- for any of the above $\sigma$ strategy only one single structure changing rule is allowed. 
Remark 1 For a kP system of degree $n$, as in Definition 2 , an $n$-tuple $\left(x_{1}, \ldots\right.$, $x_{n}$ ), where $x_{i} \in A, 1 \leq i \leq n$, are multisets over $A$, is called a configuration of $k \Pi$.

Remark 2 A computation, as usual in membrane computing, is defined as a sequence of finite steps starting from the initial configuration, with the initial multisets distributed in compartments - Definition 1 and Definition 2. In each step the rules are selected according to the execution strategy in each compartment. The result of a computation will be the number of objects collected in the output compartment. For a $\mathrm{kP}$ system, $k \Pi$, the set of all these numbers will be denoted by $N(k \Pi)$.

$P$ colony [5] represents a simple membrane system model with communities of cells communicating with a shared environment. Here we consider a restricted version of it, called $P$ colony without checking rules. More on this model can be found in [15]. The other $\mathrm{P}$ colony models will be also simplified versions of the original models, however all of them are computationally complete. We use these simplified models as we are interested in establishing connections with kernel $\mathrm{P}$ systems and prefer to use the simplest $\mathrm{P}$ colony models that are computationally complete.

Definition 7 A $P$ colony (without checking rules) is an $n+3$-tuple, $n \geq 1$

$$
\Pi=\left(O, e, F, B_{1}, \ldots, B_{n}\right),
$$

where

- $O$ is a finite set, called the alphabet of objects;

$-e \in O$ is the environment object;

$-F \subseteq O$ is the set of final objects;

- $B_{i}, 1 \leq i \leq n$, is called a cell of $\Pi$ and $B_{i}=\left(o_{i}, P_{i}\right)$, where $o_{i}$ is a multiset over $\{e\}$, called the initial multiset of the cell and $P_{i}$ is a finite set of programs, $P_{i}=\left\{p_{i, 1}, \ldots, p_{i, k_{i}}\right\}$. Each program, $p_{i, j}, 1 \leq j \leq k_{i}$, consists of a finite multiset of rules of the following forms:

- (a) $a \rightarrow b, a, b \in O$, is called internal point mutation or evolution, specifying that an object $a$ inside the cell $B_{i}$ is changed to $b$;

- (b) $c \leftrightarrow d, c, d \in O$, is called one object exchange with the environment or communication, specifying that if $c$ is contained inside the cell $B_{i}$ and $d$ is present in the environment, then $c$ is sent out of the cell into the environment while $d$ is brought inside the cell from the environment.

The multisets $o_{i}, 1 \leq i \leq n$, have the same cardinality, called capacity of $\Pi$. The number of rules in each program $p_{i, j}, 1 \leq i \leq n, 1 \leq j \leq k_{i}$, coincides with the capacity of $\Pi$.

An $n+1$-tuple $\left(x_{E}, x_{1}, \ldots, x_{n}\right)$, where $x_{E} \in(O \backslash\{e\})^{*}, x_{i} \in O^{*}$ are finite multisets, is called a configuration of $\Pi$. At the initial configuration, the environment contains arbitrarily many copies of $e$ and each cell contains inside as many objects $e$ as the capacity of $\Pi$. 
The $\mathrm{P}$ colony works with direct changes of its configurations, called transitions. To obtain a new configuration by a transition, the programs of the cells are used in the non-deterministic maximally parallel manner, i.e., each cell which is able to use one of its programs should use one. The use of a program means the parallel application of the rule(s) of the program to the object(s) inside the cell. A sequence of transitions starting from the initial configuration is a computation. A computation is successful if it is halting, i.e., if a configuration is obtained where no cell can use any program. The result of a successful computation is the number of copies of objects from $F$ present in the halting configuration. The set of numbers obtained as results of successful computations of a $\mathrm{P}$ colony $\Pi$ is denoted by $N(\Pi)$.

We introduce now the concept of $P$ colonies with senders and consumers. The definition appears for the first time in [4].

Definition 8 A P colony with senders and consumers, $\Pi$, is a $\mathrm{P}$ colony, as in Definition 7, with the following constraints:

- each cell $B_{i}, 1 \leq i \leq n$, contains always 2 objects (the capacity of $\Pi$ is 2 );

- each program $p_{i, j}, 1 \leq i \leq n, 1 \leq j \leq k_{i}$, from cell $B_{i}$ is one of the following two types:

- deletion or consumer program $\left\langle a_{i n} ; b c \rightarrow d\right\rangle$, with $a, b, c, d \in O$ and the meaning that object $a$ from the environment is consumed, i.e., it is brought into cell $B_{i}$ and the objects $b, c$ are transformed into $d$ inside of $B_{i}$;

- insertion or sender program $<a_{\text {out }} ; b \rightarrow c d>$, with $a, b, c, d \in O$ and the meaning that object $a$ is sent out of $B_{i}$ into the environment and $b$ is transformed into objects $c$ and $d$ inside of $B_{i}$.

- every program $p_{i, j}, 1 \leq i \leq n, 1 \leq j \leq k_{i}$, of cell $B_{i}$ is of the same type. A cell with only insertion programs is called a sender and a cell with only deletion programs is called a consumer.

In this model the Turing completeness is obtained for $\mathrm{P}$ colonies whereby each cell has only either consumer or producer programs. So, the cells are either consumers or senders.

Next we provide the notion of a $P$ colony with evolving environment for details regarding this computational model we refer to [2]. In the basic model the environment does not change under functioning, it can be altered only by the activity of the cells. However, to extend the concept of $\mathrm{P}$ colonies with a dynamically evolving environment is a natural idea. In this case the environment has its own rules for evolution which do not depend on the cells.

These rules are given as multiset rewriting rules, usually given as a multiset OL scheme. A multiset 0L scheme is a pair $(V, P)$, where $V$ is the alphabet of $0 \mathrm{~L}$ scheme and $P$ is a complete set of context-free multiset rewriting rules over $V$, i.e., where $P$ is a finite set of multiset rewriting rules over $V$ of the form $a \rightarrow w$, where $a \in V$ and $w \in V^{*}$ and for each $a \in V$ there exists at least one rule in $P$. For $w_{1}, w_{2} \in V^{*}$, we write $w_{1} \Rightarrow w_{2}$ if $w_{1}=a_{1} a_{2} \ldots a_{n}$ and $w_{2}=\alpha_{1} \alpha_{2} \ldots \alpha_{n}$, for $a_{i} \rightarrow \alpha_{i} \in P, 1 \leq i \leq n$, i.e., $w_{1}$ derives $w_{2}$ in one step (directly). 
Before providing the definition, we note that in the literature $\mathrm{P}$ colonies with evolving environment are also called generalized $\mathrm{P}$ colonies. In this paper, to emphasize the difference between standard $\mathrm{P}$ colonies and these developed versions, we will use the term $\mathrm{P}$ colony with evolving environment and we consider the case when the capacity of the $\mathrm{P}$ colony is 2 ..

Definition 9 A $\mathrm{P}$ colony $\Pi=\left(O, e, F, o_{E}, D_{E}, B_{1}, \ldots, B_{n}\right)$ with evolving environment is a combination of the concept of a $\mathrm{P}$ colony in Definition 7 and the concepts of a $\mathrm{P}$ colony with senders and consumers in Definition 8 with the following additional constraints:

$-o_{E} \in(O \backslash\{e\})^{*}$,

- $D_{E}=\left(O \backslash\{e\}, P_{E}\right)$ is a multiset $0 L$ scheme,

- each cell $B_{i}, 1 \leq i \leq n$, contains always 2 objects (the capacity of $\Pi$ is 2 ); each program $p_{i, j}, 1 \leq i \leq n, 1 \leq j \leq k_{i}$ of $B_{i}$ has a finite set of programs of exactly one of the following three types:

- deletion or consumer program $\left\langle a_{i n} ; b c \rightarrow d\right\rangle$, with $a, b, c, d \in O$ and the meaning that object $a$ from the environment is consumed, i.e., it is brought into cell $B_{i}$ and the objects $b, c$ are transformed into $d$ inside of $B_{i}$;

- insertion or sender program $<a_{\text {out }} ; b \rightarrow c d>$, with $a, b, c, d \in O$ and the meaning that object $a$ is sent out of $B_{i}$ into the environment and $b$ is transformed into objects $c$ and $d$ inside of $B_{i}$.

- a program with two rules which are evolution rules (of the form $<a \rightarrow$ $b>$ ) and/or communication rules (of the form $<a \leftrightarrow b>$ ), as given in Definition 7.

The initial configuration of a $\mathrm{P}$ colony with evolving environment is the $(n+1)$-tuple $\left(o_{E}, o_{1}, \ldots, o_{n}\right), o_{i}, 1 \leq i \leq n$, as given in Definition 7 .

By applying programs, the $\mathrm{P}$ colony with evolving environment passes from one configuration to some other configuration. Those objects in the environment which are not affected by any program in the given step are rewritten by the multiset $0 \mathrm{~L}$ scheme $D_{E}$. As in the previous cases, the systems works with the non-deterministic maximally parallel manner, i.e., at each step a maximal number of cells performs one of its programs in parallel (the program is chosen non-deterministically out of the applicable ones). Those objects in the environment which are different from the basic object, $e$, and are not affected by any program, are changed according to the rules of the multiset $0 L$ scheme.

A computation is a sequence of consecutive configurations starting from the initial configuration and ending in a configuration when no cell has any applicable program. Notice that in this case the work of the $\mathrm{P}$ colony itself does not necessarily stop, since the multiset $0 L$ scheme may continue changing the non-environment objects in the environment, if such an object exists.

The result of the computation is the number of copies of final objects in the environment in such a configuration when no cell is able to perform at least one program. 


\section{Relationships between various $\mathbf{P}$ colonies and kernel $\mathbf{P}$ systems}

We start by looking at the relationships between $\mathrm{P}$ colonies of capacity $h$, $h \geq 1$, and $\mathrm{kP}$ systems. It may be observed from the proofs of these results that we use $\mathrm{kP}$ systems with only rewriting and communication rules. Guards are only used in the last theorem.

Theorem 1 For every $P$ colony $\Pi=\left(O, e, F, B_{1}, \ldots, B_{n}\right), n \geq 1$, of capacity $h, h \geq 1$, one can construct a $k P$ system $k \Pi=\left(A, \mu, C_{1}, C_{2}, 2\right)$, such that $N(\Pi)=N(k \Pi)$ holds.

Proof Let us consider $\Pi=\left(O, e, F, B_{1}, \ldots, B_{n}\right), n \geq 1$, a $\mathrm{P}$ colony of size $n$ and capacity $h$. It is known that the result of a computation in $\Pi$ is obtained in the environment, consisting only of objects from $F$. The cell $B_{i}, 1 \leq i \leq n$, has the initial multiset $o_{i}$ consisting of $h$ objects and each program $p_{i, j}, 1 \leq j \leq k_{i}$, from the set of programs, $P_{i}$, consists of $h$ rules.

We will build a $\mathrm{kP}$ system with two compartments

$$
k \Pi=\left(A, \mu, C_{1}, C_{2}, 2\right)
$$

where $C_{1}$ is simulating the behaviour of the $\mathrm{P}$ colony, $\Pi$, and $C_{2}$ is collecting the result that coincides with the result of the $\mathrm{P}$ colony, $\Pi$.

The objects of the $\mathrm{kP}$ system are $A=F \cup\left\{f^{\prime} \mid f \in F\right\} \cup\{[a, i] \mid a \in O, 0 \leq i \leq$ $n\}$. For each $a \in O$, when it belongs to cell $B_{i}, 1 \leq i \leq n$, or the environment, 0 , it will be denoted by $[a, i]$ or $[a, 0]$, respectively.

The membrane structure, $\mu$, provides a connection between the two compartments, $C_{1}$ and $C_{2}$, allowing them to exchange objects, where $C_{i}=\left(t_{i}, w_{i}\right)$, $i=1,2$. Each $t_{i}, 1 \leq i \leq 2$, the type of compartment $C_{i}$, is given by $t_{i}=\left(R_{i}, \sigma_{i}\right)$, where $R_{i}$ is the set of rules and $\sigma_{i}$ the execution strategy. These will be introduced later on. The second component of $C_{i}$ is the initial multiset of the compartment. We have $w_{1}=\left[o_{1}, 1\right] \ldots\left[o_{n}, n\right]$ and $w_{2}=\lambda$.

The type, $t_{1}$, of the compartment $C_{1}$ has the set of rules, $R_{1}$, given below.

Next we define the rules of $k \Pi$. This will be done in several steps. Notice first that any program $p_{i, j} \in P_{i}, 1 \leq i \leq n, 1 \leq j \leq k_{i}$, consists of $h$ rules $r_{i, j, k}$, $1 \leq k \leq h$ that can be evolution rules or communication rules. Furthermore, if a program is applied, then all of its rules should be performed in parallel.

First we will define rules $r_{i, j, k}^{\prime}$ to rules $r_{i, j, k}$ in program $p_{i, j} \in P_{i}, 1 \leq i \leq n$, $1 \leq j \leq k_{i}, l \leq k \leq h$. When

1. $r_{i, j, k}: a \rightarrow b$ with $a, b \in O$, then let $r_{i, j, k}^{\prime}:[a, i] \rightarrow[b, i]$.

2. $r_{i, j, k}: c \leftrightarrow d$ with $c, d \in(O \backslash(F \cup\{e\}))$, then let $r_{i, j, k}^{\prime}:[c, i][d, 0] \rightarrow$ $[c, 0][d, i]$.

3. $r_{i, j, k}: c \leftrightarrow e$ with $c \in(O \backslash(F \cup\{e\}))$, then let $r_{i, j, k}^{\prime}:[c, i] \rightarrow[c, 0][e, i]$.

4. $r_{i, j, k}: e \leftrightarrow d$ with $d \in(O \backslash(F \cup\{e\}))$, then let $r_{i, j, k}^{\prime}:[e, i][d, 0] \rightarrow[d, i]$

5. $r_{i, j, k}: e \leftrightarrow e$ then let $r_{i, j, k}^{\prime}:[e, i] \rightarrow[e, i]$.

6. $r_{i, j, k}: f \leftrightarrow d$ with $d \in(O \backslash(F \cup\{e\})), f \in F$, then let $r_{i, j, k}^{\prime}:[f, i][d, 0] \rightarrow$ $[f, 0][d, i]\left(f, t_{2}\right)$, where $t_{2}$ is the type of $C_{2}$ - its rules will be given later. In this case $f, f \in F$, is also sent to $C_{2}$. 
7. $r_{i, j, k}: c \leftrightarrow f$ with $c \in(O \backslash(F \cup\{e\})), f \in F$, then let $r_{i, j, k}^{\prime}:[c, i][f, 0] \rightarrow$ $[c, 0][f, i]\left(f^{\prime}, t_{2}\right)$, where $t_{2}$ is as above. In this case $f^{\prime}, f \in F$, is sent to $C_{2}$.

8. $r_{i, j, k}: f_{1} \leftrightarrow f_{2}$ with $f_{1}, f_{2} \in F$, then let $r_{i, j, k}^{\prime}:\left[f_{1}, i\right]\left[f_{2}, 0\right] \rightarrow\left[f_{1}, 0\right]\left[f_{2}, i\right]\left(f_{1}, t_{2}\right)\left(f_{2}^{\prime}, t_{2}\right)$.

9. $r_{i, j, k}: f \leftrightarrow e$ with $f \in F$, then let $r_{i, j, k}^{\prime}:[f, i] \rightarrow[f, 0][e, i]\left(f, t_{2}\right)$.

10. $r_{i, j, k}: e \leftrightarrow f$ with $f \in F$, then let $r_{i, j, k}^{\prime}:[e, i][f, 0] \rightarrow[f, i]\left(f^{\prime}, t_{2}\right)$.

The set of rules of $R_{2}$ associated with type $t_{2}$ contains rules $p_{f}: f f^{\prime} \rightarrow \lambda$, $f \in F$.

Each rule $r_{i, j, k}, 1 \leq i \leq n, 1 \leq j \leq k_{i}, 1 \leq k \leq h$, interacts with two objects - either both from the cell $B_{i}$, when $r_{i, j, k}$ is an evolution rule, or one from $B_{i}$ and the other from the environment, when it is a communication rule. Cases (1) and (2) above correspond to evolution rules and communication rules, respectively. For a rule $r_{i, j, k}, 1 \leq i \leq n, 1 \leq j \leq k_{i}, 1 \leq k \leq h$, interacting with objects $a, b$ from $B_{i}$, the corresponding rule $r_{i, j, k}^{\prime}$, transforms $[a, i]$ into $[b, i]$. When the rule interacts with an object $a$ from $B_{i}$ and $b$ from environment then the corresponding rule rewrites $[a, i]$ and $[b, 0]$ into $[a, 0],[b, i]$. Cases $(3)$ to (10) present special cases of (2) when the objects are $e$ or from $F$. When object $e$ refers to an occurrence within the environment $(3-5,9)$ then the corresponding object in $r_{i, j, k}^{\prime}$ does not appear, i.e., $[e, 0]$ is not present in any of these rules. When an object $f, f \in F$, is sent to the environment $(6,8,9)$ then the corresponding rules from $R_{1}$ have on the right hand side $\left(f, t_{2}\right)$, which means that $f$ is also sent to $C_{2}$, the output compartment. When $f, f \in F$, is brought into $B_{i}$ from environment $(7,810)$ then the rules from $R_{1}$ have on the right hand side $\left(f^{\prime}, t_{2}\right), f \in F$; this means that $f^{\prime}$ is sent to $C_{2}$ and this will be used to erase $f$ by using the rule $r_{f}$ from $R_{2}$.

Since every program of $\Pi$ consists of exactly $h$ rules, we describe how the the rules of $R_{1}$ are constructed that correspond to the programs of $\Pi$ with only evolution or communication rules.

We first need an auxiliary notation. For a rule of the form $r: u \rightarrow v$, where $u, v \in V^{*}$, where $V$ is an alphabet, $u$ and $v$ are finite multisets over $V$, we define $l h s(r)=u$ and $r h s(r)=v$.

For each program $p_{i, j}=<r_{i, j, 1} \ldots, r_{i, j, h}>\in P_{i}, 1 \leq j \leq k_{i}, 1 \leq i \leq n$, the set $R_{1}$ contains a rule of the form

$$
p_{i, j}^{\prime}: u_{i, j} \rightarrow v_{i, j},
$$

where $u_{i, j}=\operatorname{lhs}\left(r_{i, j, 1}^{\prime}\right) \ldots \operatorname{lhs}\left(r_{i, j, h}^{\prime}\right)$ and $v_{i, j}=r h s\left(r_{i, j, 1}^{\prime}\right) \ldots r h s\left(r_{i, j, h}^{\prime}\right)$, and $r_{i, j, k}^{\prime} 1 \leq i \leq n, 1 \leq j \leq k_{i}, 1 \leq k \leq h$, is associated to $r_{i, j, h}$ in accordance with one of the cases (1) - (10) above. (Note that $u_{i, j}$ and $v_{i, j}$ are strings representing finite multisets of objects).

The execution strategies of the types $t_{1}, t_{2}$ are given by $\sigma_{1}=\left\{p_{i, j}^{\prime} \mid 1 \leq i \leq\right.$ $\left.n, 1 \leq j \leq k_{i}\right\}^{\top}$ and $\sigma_{2}=\left\{p_{f} \mid f \in F\right\}^{\top}$. This means that each compartment executes the rules using maximal parallelism mode.

A computation in $\Pi$ starts from the initial configuration $\left(x_{E}, x_{1}, \ldots, x_{n}\right)$ where $x_{E}=\lambda$ and $x_{i}=e^{h}, 1 \leq i \leq n$. The initial configuration of $k \Pi$ is $\left(\left[e^{h}, 1\right] \ldots\left[e^{h}, n\right], \lambda\right)$, where $\left[e^{h}, i\right], 1 \leq i \leq n$, denotes $h$ objects $[e, i]$. In 
each cell $B_{i}, 1 \leq i \leq n$, at most one program, consisting of $h$ rules, can be applied, as $B_{i}$ has always $h$ objects from $O$. Similarly, in $k \Pi$ there are always $h$ objects of the form $[a, i]$, where $a \in O$. For any configuration of $\Pi$, $\left(x_{E}, x_{1}, \ldots, x_{n}\right)$, where $x_{E}$ is a multiset over $O \backslash\{e\}$ and $x_{i}, 1 \leq i \leq n$, is a multiset over $O$ with $h$ objects. The corresponding configuration of $k \Pi$ is $\left(\left[x_{E}, 0\right]\left[x_{1}, 1\right] \ldots\left[x_{n}, n\right], y\right)$, where $\left[x_{i}, i\right], 1 \leq i \leq n$, denotes $h$ objects $[a, i]$, for each $a$ occurring in $x_{i}$ (similarly for $\left[x_{E}, 0\right]$ ). A program $p_{i, j}, 1 \leq i \leq n$, $1 \leq j \leq k_{i}$, is applied in $B_{i}$ of $\Pi$ if and only if $p_{i, j}^{\prime}$ is applied in $C_{1}$ of $k \Pi$. Hence $x_{i}, 1 \leq i \leq n$, appears in a configuration of $\Pi$ if and only if $\left[x_{i}, i\right]$ appears in the corresponding configuration of $k \Pi$. The multiset $x_{E}$ appears in the environment of $\Pi$ if and only if $\left[x_{E}, 0\right]$ appears in $k \Pi$. The multiset $y$ is given by $y=x_{E} u u^{\prime}$, where $u=f_{1} \ldots f_{p}$ and $u=f_{1}^{\prime} \ldots f_{p}^{\prime}$. The multiset $u u^{\prime}$ is erased in the next step by applying rules of $R_{2}$ in a maximally parallel manner.

These show that the two mechanisms arrive at the same number of objects from $F$ in the environment $(\mathrm{P}$ colony $\Pi)$ and in $C_{2}(\mathrm{kP}$ system $k \Pi)$ in a halting computation, hence $N(\Pi)=N(k \Pi)$.

Now we are looking at $\mathrm{P}$ colonies with senders and consumers, aiming at simulating them by using again $\mathrm{kP}$ systems.

Theorem 2 For every $P$ colony $\Pi=\left(O, e, F, B_{1}, \ldots, B_{n}\right), n \geq 1$, with senders and consumers, one can construct a $k P$ system $k \Pi=\left(A, \mu, C_{1}, C_{2}, 2\right)$, such that $N(\Pi)=N(k \Pi)$ holds.

Proof In this proof we follow similar ideas as in the proof of Theorem 1. Let us consider $\Pi=\left(O, e, F, B_{1}, \ldots, B_{n}\right), n \geq 1$, a $\mathrm{P}$ colony with senders and consumers of size $n$ and capacity 2. The result of a computation in $\Pi$ is obtained in the environment, as the number of objects from $F$. The cell $B_{i}, 1 \leq i \leq n$, has the initial multiset $o_{i}$ consisting of two objects and each program $p_{i, j}, 1 \leq j \leq k_{i}$, from the set of programs, $P_{i}$, is either a sender or a consumer. So, we have either consumer or producer cells.

We build, similar to the proof of Theorem 1, a $\mathrm{kP}$ system with two compartments

$$
k \Pi=\left(A, \mu, C_{1}, C_{2}, 2\right)
$$

where $\mu, C_{1}$ and $C_{2}$ have the same meaning and $A$ the same definition,

$$
A=F \cup\left\{f^{\prime} \mid f \in F\right\} \cup\{[a, i] \mid a \in O, 0 \leq i \leq n\},
$$

as in the proof of Theorem 1.

Each compartment, $C_{i}, i=1,2$, has a type, $t_{i}$, and an initial multiset $w_{i}$. These multisets are $w_{1}=\left[o_{1}, 1\right] \ldots\left[o_{n}, n\right]$ and $w_{2}=\lambda$. Each $o_{i}, 1 \leq i \leq n$, consists of two objects, $o_{i}=a b$. In this case $\left[o_{i}, i\right]$ means $[a, i][b, i]$.

In the sequel we define the set of rules $R_{1}$ and $R_{2}$, from compartments $C_{1}$ and $C_{2}$, respectively. For an arbitrary program $p_{i, j} \in P_{i}, 1 \leq i \leq n$, $1 \leq j \leq k_{i}$, we will associate a rule $p_{i, j}^{\prime} \in R_{1}$. As these programs are either senders or consumers, we will analyse each of these situations below. When 
1. $p_{i, j}:<a_{\text {out }} ; b \rightarrow c d>$ (sender) with $a, b, c, d \in O, a \notin F, a \neq e$, then $R_{1}$ has a rule $p_{i, j}^{\prime}:[a, i][b, i] \rightarrow[a, 0][c, i][d, i]$.

2. $p_{i, j}:<f_{\text {out }} ; b \rightarrow c d>$ (sender) with $f, b, c, d \in O, f \in F$, then $R_{1}$ has a rule $p_{i, j}^{\prime}:[f, i][b, i] \rightarrow[f, 0][c, i][d, i]\left(f, t_{2}\right)$, where $t_{2}$ is the type of $C_{2}$ - its rules will be given later. In this case $f, f \in F$, is also sent to $C_{2}$.

3. $p_{i, j}:<e_{\text {out }} ; b \rightarrow c d>$ (sender) with $b, c, d \in O$, then $R_{1}$ has a rule $p_{i, j}^{\prime}:$ $[e, i][b, i] \rightarrow[c, i][d, i]$.

4. $p_{i, j}:<a_{i n} ; b c \rightarrow d>$ (consumer) with $a, b, c, d \in O, a \notin F, a \neq e$, then $R_{1}$ has a rule $p_{i, j}^{\prime}:[a, 0][b, i][c, i] \rightarrow[a, i][d, i]$.

5. $p_{i, j}:<f_{i n} ; b c \rightarrow d>$ (consumer) with $b, c, d \in O, f \in F$, then $R_{1}$ has a rule $p_{i, j}^{\prime}:[f, 0][b, i][c, i] \rightarrow[f, i][d, i]\left(f^{\prime}, t_{2}\right)$. In this case $f^{\prime}, f \in F$, is sent to $C_{2}$.

6. $p_{i, j}:<e_{i n} ; b c \rightarrow d>$ (consumer) with $b, c, d \in O$, then $R_{1}$ has a rule $p_{i, j}^{\prime}:[b, i][c, i] \rightarrow[e, i][d, i]$.

The set of rules $R_{2}$ associated with type $t_{2}$ contains rules $p_{f}: f f^{\prime} \rightarrow \lambda, f \in F$.

Each program $p_{i, j} \in P_{i}, 1 \leq i \leq n, 1 \leq j \leq k_{i}$, includes a rule interacting only with objects, $b, c, d$, from the cell $B_{i}$. The corresponding rule $p_{i, j}^{\prime}, 1 \leq i \leq$ $n, 1 \leq j \leq k_{i}$, cases (1-6), includes $[b, i],[c, i],[d, i]$, respectively. The other rule that appears in each program $p_{i, j} \in P_{i}, 1 \leq i \leq n, 1 \leq j \leq k_{i}$, either sends an $a$ to the environment (1) or consumes it from the environment (4). In these cases the corresponding rule $p_{i, j}^{\prime}, 1 \leq i \leq n, 1 \leq j \leq k_{i}$, transforms $[a, i]$ into $[a, 0](1)$ or the other way around (4). When $a=f, f \in F$, then the rule $p_{i, j}^{\prime}$, $1 \leq i \leq n, 1 \leq j \leq k_{i}$, corresponding to the sender (2) includes on the right hand side $\left(f, t_{2}\right)$, indicating that $f$ is also sent to $C_{2}$, the output compartment. When $f, f \in F$, is brought into $B_{i}$ from environment, the consumer, then the corresponding rule $p_{i, j}^{\prime}, 1 \leq i \leq n, 1 \leq j \leq k_{i}$, (5) has on the right hand side $\left(f^{\prime}, t_{2}\right), f \in F$; this means that $f^{\prime}$ is sent to $C_{2}$ and this will be used to erase $f$ by using the rule $p_{f}$ from $R_{2}$. When object $e$ refers to an occurrence within the environment $(3,6)$ then the corresponding object in $p_{i, j}^{\prime}, 1 \leq i \leq n$, $1 \leq j \leq k_{i}$, does not appear, i.e., $[e, 0]$ is not present in any of these rules.

The execution strategies of the types $t_{1}, t_{2}$, as in the proof of Theorem 1 , are given by $\sigma_{1}=\left\{p_{i, j}^{\prime} \mid 1 \leq i \leq n, 1 \leq j \leq k_{i}\right\}^{\top}$ and $\sigma_{2}=\left\{p_{f} \mid f \in F\right\}^{\top}$, i.e., in each compartment the rules are executed using maximal parallelism mode.

A computation in $\Pi$ starts from the initial configuration $\left(x_{E}, x_{1}, \ldots, x_{n}\right)$ where $x_{E}=\lambda$ and $x_{i}=e e, 1 \leq i \leq n$. The initial configuration of $k \Pi$ is $([e, 1][e, 1] \ldots[e, n][e, n], \lambda)$. In each cell $B_{i}, 1 \leq i \leq n$, at most one program, consisting of either a sender or a consumer, can be applied, as $B_{i}$ has always two objects $a, b$. Similarly, in $k \Pi$ there are always two object $[a, i],[b, i]$. For any configuration of $\Pi,\left(x_{E}, x_{1}, \ldots, x_{n}\right)$, where $x_{E}$ is a multiset over $O \backslash\{e\}$ and $x_{i}, 1 \leq i \leq n$, is a multiset over $O$ with two objects. The corresponding configuration of $k \Pi$ is $\left(\left[x_{1}, 1\right] \ldots\left[x_{n}, n\right], y\right)$. A program $p_{i, j}, 1 \leq i \leq n, 1 \leq j \leq$ $k_{i}$, is applied in $B_{i}$ of $\Pi$ if and only if $p_{i, j}^{\prime}$ is applied in $C_{1}$ of $k \Pi$. Hence $x_{i}$, $1 \leq i \leq n$, appears in a configuration of $\Pi$ if and only if $\left[x_{i}, i\right]$ appears in the corresponding configuration of $k \Pi$. The multiset $y$ is given by $x_{E}$ and maybe 
a multiset $u u^{\prime}$, where $u=f_{1} \ldots f_{p}$ and $u=f_{1}^{\prime} \ldots f_{p}^{\prime}$. The multiset $u u^{\prime}$ is erased in the next step by applying rules of $R_{2}$ in a maximal parallel manner.

These show that the two mechanisms arrive at the same number of objects from $F$ in the environment $(\mathrm{P}$ colony $\Pi)$ and in $C_{2}(\mathrm{kP}$ system $k \Pi)$ in a halting computation, hence $N(\Pi)=N(k \Pi)$.

Next we show that kernel $\mathrm{P}$ systems simulate $\mathrm{P}$ colonies with evolving environment as well. Notice that in the case of these $\mathrm{P}$ colony variants the possibly dynamically changing environment is expected to alter the computational power of the $\mathrm{P}$ colony.

Theorem 3 For every $P$ colony $\Pi=\left(O, e, F, o_{E}, D_{E}, B_{1}, \ldots, B_{n}\right), n \geq 1$, with evolving environment and with capacity 2 one can construct a $k P$ system $k \Pi=\left(A, \mu, C_{1}, C_{2}, 2\right)$, such that $N(\Pi)=N(k \Pi)$ holds

Proof Let us consider $\Pi=\left(O, e, F, o_{E}, D_{E}, B_{1}, \ldots, B_{n}\right), n \geq 1$, a $\mathrm{P}$ colony of size $n$ and capacity 2 . Without the loss of generality, we may assume that cells $B_{i} 1 \leq m \leq n$ are of type sender and consumer ( $m$ is an even number and $\Pi$ has as many sender cells as consumer cells) and cells $B_{j}, m+1 \leq j \leq n$ have only evolution and/or communication rules.

The cell $B_{i}, 1 \leq i \leq n$, has the initial multiset, $o_{i}$, consisting of two objects and each program, $p_{i, j}, 1 \leq j \leq k_{i}$, in the set of programs, $P_{i}$, depending of the type of $B_{i}$ consists of one or two rules.

To prove the statement, we construct a $\mathrm{kP}$ system with two compartments

$$
k \Pi=\left(A, \mu, C_{1}, C_{2}, 2\right)
$$

where $C_{1}$ simulates the computations of $\Pi$ and $C_{2}$ collects the result of $k \Pi$ that is equal to the result of $\Pi$.

The objects of the $\mathrm{kP}$ system are $A=F \cup\left\{f^{\prime} \mid f \in F\right\} \cup\{[a, i] \mid a \in O, 0 \leq$ $i \leq n\}$, as in the case of the proofs of Theorem 1 and Theorem 2. Each $a \in O$, when it appears in cell $B_{i}, 1 \leq i \leq n$, or in the the environment, 0 , it will be denoted by $[a, i], 1 \leq i \leq n$, or $[a, 0]$, respectively.

The membrane structure, $\mu$, provides a connection between the two compartments, $C_{1}$ and $C_{2}$, allowing them to exchange objects, where $C_{i}=\left(t_{i}, w_{i}\right)$, $i=1,2$. Each $t_{i}, 1 \leq i \leq 2$, the type of compartment $C_{i}$, is given by $t_{i}=\left(R_{i}, \sigma_{i}\right)$, where $R_{i}$ is the set of rules and $\sigma_{i}$ the execution strategy that are defined analogously to the previous proofs, with slight changes for $\sigma_{1}$ as it will be shown when this execution strategy is defined. The second component of $C_{i}$, the initial multiset of the compartment, is given as $w_{1}=\left[o_{1}, 1\right] \ldots\left[o_{n}, n\right]$, where each $o_{i}, 1 \leq i \leq n$, consists of two objects $a, b$ and $\left[o_{i}, i\right]$ denotes $[a, i][b, i]$, and $w_{2}=\lambda$. We will look at these initial multisets when the behaviour of $k \Pi$ will be discussed.

As in the previous proofs the compartment $C_{1}$ will host the objects and rules corresponding to those of $\Pi$ and $C_{2}$ will collect the results of the computation. In this case the simulation of the $\mathrm{P}$ colony $\Pi$ in $C_{1}$ will be such that in each of the computation step in $\Pi$ are first applied the programs in 
cells $B_{i}, 1 \leq i \leq n$, and if there is at least one cell with at least an applicable program then the $0 L$ scheme $D_{E}$ is also used. This two stage application of the programs followed by the use of the $0 L$ scheme $D_{E}$ will be simulated in $C_{1}$ by a special definition of the execution strategy $\sigma_{1}$.

We define the rule sets $R_{1}, R_{2}$ of $k \Pi$ in several steps.

We start by defining rule set $R_{1}^{\prime}$, a subset of $R_{1}$, which is associated with the type $t_{1}$ of $C_{1}$ that corresponds to the programs of cells $B_{i}, 1 \leq i \leq m$, where $m \leq n$. The reader may easily notice that these rules can be obtained exactly in the same manner as the rules were contructed in the proof of Theorem 2, in case of $\mathrm{P}$ colonies with senders and consumers. Thus, we define for an arbitrary program $p_{i, j} \in P_{i}, 1 \leq i \leq m, 1 \leq j \leq k_{i}$, a rule $p_{i, j}^{\prime} \in R_{1}^{\prime}$ as follows:

1. $p_{i, j}:<a_{\text {out }} ; b \rightarrow c d>$ with $a, b, c, d \in O, a \notin F, a \neq e$, then $R_{1}^{\prime}$ has a rule $p_{i, j}^{\prime}:[a, i][b, i] \rightarrow[a, 0][c, i][d, i]$.

2. ${ }^{\prime} p_{i, j}:<f_{\text {out }} ; b \rightarrow c d>$ with $f, b, c, d \in O, f \in F$, then $R_{1}^{\prime}$ has a rule $p_{i, j}^{\prime}:[f, i][b, i] \rightarrow[f, 0][c, i][d, i]\left(f, t_{2}\right)$, where $t_{2}$ is the type of $C_{2}-$ as in the previous prrofs, its rules will be given later. In this case $f, f \in F$, is also sent to $C_{2}$.

3. $p_{i, j}:<e_{\text {out }} ; b \rightarrow c d>$ with $b, c, d \in O$, then $R_{1}^{\prime}$ has a rule $p_{i, j}^{\prime}:[e, i][b, i] \rightarrow$ $[c, i][d, i]$.

4. $p_{i, j}:<a_{i n} ; b c \rightarrow d>$ with $a, b, c, d \in O, a \notin F, a \neq e$, then $R_{1}^{\prime}$ has a rule $p_{i, j}^{\prime}:[a, 0][b, i][c, i] \rightarrow[a, i][d, i]$.

5. $p_{i, j}:<f_{i n} ; b c \rightarrow d>$ with $b, c, d \in O, f \in F$, then $R_{1}^{\prime}$ has a rule $p_{i, j}^{\prime}$ : $[f, 0][b, i][c, i] \rightarrow[f, i][d, i]\left(f^{\prime}, t_{2}\right)$. In this case $f^{\prime}, f \in F$, is sent to $C_{2}$.

6. $p_{i, j}:<e_{i n} ; b c \rightarrow d>$ with $b, c, d \in O$, then $R_{1}^{\prime}$ has a rule $p_{i, j}^{\prime}:[b, i][c, i] \rightarrow$ $[e, i][d, i]$.

The correspondence of the above rules of $k \Pi$ and the programs of cells $B_{i}$, $1 \leq i \leq m$, and their role in the computation can easily be obtained by the same reasoning as we used in the proof of Theorem 2 .

Next we define the rule set $R_{1}^{\prime \prime}$, a subset of $R_{1}$, which is associated with the type $t_{1}$ of $C_{1}$ that corresponds to the programs of cells $B_{i}, m+1 \leq i \leq n$. Notice first that any program $p_{i, j} \in P_{i}, m+1 \leq i \leq n, 1 \leq j \leq k_{i}$, consists of two rules $r_{i, j, 1}$ and $r_{i, j, 2}$, where every rule can be an evolution rule or a communication rule. Furthermore, if a program is applied, then both rules should be performed in parallel.

Analogously to the proof of Theorem 1, first we will define rules $r_{i, j, k}^{\prime}$ corresponding to rules $r_{i, j, k}$ from programs $p_{i, j} \in P_{i}, 1 \leq i \leq n, 1 \leq j \leq k_{i}$, $k=1,2$. When

1. $r_{i, j, k}: a \rightarrow b$ with $a, b \in O$, then let $r_{i, j, k}^{\prime}:[a, i] \rightarrow[b, i]$.

2. $r_{i, j, k}: c \leftrightarrow d$ with $c, d \in(O \backslash(F \cup\{e\}))$, then let $r_{i, j, k}^{\prime}:[c, i][d, 0] \rightarrow$ $[c, 0][d, i]$.

3. $r_{i, j, k}: c \leftrightarrow e$ with $c \in(O \backslash(F \cup\{e\}))$, then let $r_{i, j, k}^{\prime}:[c, i] \rightarrow[c, 0][e, i]$.

4. $r_{i, j, k}: e \leftrightarrow d$ with $d \in(O \backslash(F \cup\{e\}))$, then let $r_{i, j, k}^{\prime}:[e, i][d, 0] \rightarrow[d, i]$.

5. $r_{i, j, k}: e \leftrightarrow e$ then let $r_{i, j, k}^{\prime}:[e, i] \rightarrow[e, i]$. 
6. $r_{i, j, k}: f \leftrightarrow d$ with $d \in(O \backslash(F \cup\{e\})), f \in F$, then let $r_{i, j, k}^{\prime}:[f, i][d, 0] \rightarrow$ $[f, 0][d, i]\left(f, t_{2}\right)$, where $t_{2}$ is the type of $C_{2}$ - its rules will be given later. In this case $f, f \in F$, is also sent to $C_{2}$.

7. $r_{i, j, k}: c \leftrightarrow f$ with $c \in(O \backslash(F \cup\{e\})), f \in F$, then let $r_{i, j, k}^{\prime}:[c, i][f, 0] \rightarrow$ $[c, 0][f, i]\left(f^{\prime}, t_{2}\right)$, where $t_{2}$ is as above. In this case $f^{\prime}, f \in F$, is sent to $C_{2}$.

8. $r_{i, j, k}: f_{1} \leftrightarrow f_{2}$ with $f_{1}, f_{2} \in F$, then let

$r_{i, j, k}^{\prime}:\left[f_{1}, i\right]\left[f_{2}, 0\right] \rightarrow\left[f_{1}, 0\right]\left[f_{2}, i\right]\left(f_{1}, t_{2}\right)\left(f_{2}^{\prime}, t_{2}\right)$.

9. $r_{i, j, k}: f \leftrightarrow e$ with $f \in F$, then let $r_{i, j, k}^{\prime}:[f, i] \rightarrow[f, 0][e, i]\left(f, t_{2}\right)$.

10. $r_{i, j, k}: e \leftrightarrow f$ with $f \in F$, then let $r_{i, j, k}^{\prime}:[e, i][f, 0] \rightarrow[f, i]\left(f^{\prime}, t_{2}\right)$.

The meaning of the rules above is the same that of the corresponding rules in the proof of Theorem 1, therefore we omit the explanations. Since every program of $\Pi$ consists of exactly two rules, the rules of $k \Pi$ that correspond to the programs of $\Pi$ with evolution and/or communication rules are constructed analogously to the way that the set of rules $R_{1}$ defined in the proof of Theorem 1 , taking capacity $h=2$.

Now we define the rules that appear in $R_{1}^{\prime \prime}$.

For each program $p_{i, j}=<r_{i, j, 1}, r_{i, j, 2}>, m+1 \leq i \leq n, 1 \leq j \leq k_{i}$, from $P_{i}$ of the cell $B_{i}$, the rules $r_{i, j, 1}^{\prime}$ and $r_{i, j, 2}^{\prime}$ are built as shown above. Denoting $u_{i, j}=\operatorname{lhs}\left(r_{i, j, 1}^{\prime}\right) \operatorname{lhs}\left(r_{i, j, 2}^{\prime}\right)$ and $v_{i, j}=r h s\left(r_{i, j, 1}^{\prime}\right) r h s\left(r_{i, j, 2}^{\prime}\right)$, as in the proof of Theorem 1 , we add $p_{i, j}^{\prime}: u_{i, j} \rightarrow v_{i, j}$ to $R_{1}^{\prime \prime}$.

Next we define the rule set $R_{1}^{\prime \prime \prime}$, a subset of $R_{1}$, which simulates the rules in rule set $P_{E}$ of the multiset $0 L$ scheme $D_{E}$. For each rule of the form $r: a \rightarrow$ $u \in P_{E}$, where $a \in O$ and $u=b_{1} \ldots b_{s}, b_{l} \in O, 1 \leq l \leq s$, or $p: a \rightarrow \lambda \in P_{E}$ we assign a rule of the form $r^{\prime}:[a, 0] \rightarrow\left[b_{1}, 0\right] \ldots\left[b_{s}, 0\right]\{g\}$ or $p^{\prime}:[a, 0] \rightarrow$ $\lambda\{g\}$, respectively. The guard, defined below, will impose constraints on the application of the rules from $R_{1}^{\prime \prime \prime}$, such that these are applied if and only if the rules associated to a program from at least one cell $B_{i}, 1 \leq i \leq n$, are applicable to the current multiset. With this constraint we make sure that the rules of $R_{1}^{\prime \prime \prime}$ will stop being applied when no rule from $R_{1}^{\prime} \cup R_{1}^{\prime \prime}$ is applicable, and consequently the computation stops.

The rule set $R_{1}$ is given by $R_{1}^{\prime} \cup R_{1}^{\prime \prime} \cup R_{1}^{\prime \prime \prime}$.

In order to define the guard $g$ that appears in every rule from $R_{1}^{\prime \prime \prime}$ we introduce a notation. For a rule $r \in R_{1}^{\prime} \cup R_{1}^{\prime \prime}, l h s(r)$ is a multiset, that appears on the left hand side of the rule. If this is a multiset over $\left\{a_{1}, \ldots, a_{t}\right\}$, then it might be written as $a_{1}^{p_{1}} \ldots a_{t}^{p_{t}}$, with $p_{i} \geq 1,1 \leq i \leq t$. We introduce the guard $g_{l h s(r)}$ denoting $\geq a_{1}^{p_{1}} \wedge \cdots \wedge \geq a_{t}^{p_{t}}$. This guard is true for a multiset $w$ if and only if $w$ contains at least $p_{i}$ occurrences of $a_{i}, 1 \leq i \leq t$. Having $g_{l h s(r)}$ defined for every $r \in R_{1}^{\prime} \cup R_{1}^{\prime \prime}$, one can define the guard $g$ as follows

$$
g=g_{l h s\left(p_{1,1)}^{\prime}\right.} \vee \cdots \vee g_{l h s\left(p_{\left.1, k_{1}\right)}^{\prime}\right.} \vee \cdots \vee g_{l h s\left(p_{n, 1)}^{\prime}\right.} \vee \cdots \vee g_{l h s\left(p_{\left.n, k_{n}\right)}^{\prime}\right.} .
$$

The execution strategy of type $t_{1}$ is $\sigma_{1}=\left\{r \mid r \in R_{1}^{\prime} \cup R_{1}^{\prime \prime}\right\}^{\top}\left\{r \mid r \in R_{1}^{\prime \prime \prime}\right\}^{\top}$. This means that first are applied in maximal parallel manner the rules from $R_{1}^{\prime} \cup R_{1}^{\prime \prime}$ followed by rules from $R_{1}^{\prime \prime \prime}$ applied also in maximal parallel way.

The set of rules of $R_{2}$ associated with type $t_{2}$ contains rules $p_{f}: f f^{\prime} \rightarrow \lambda$, $f \in F$. 
The execution strategy of the type $t_{2}$ is given by $\sigma_{2}=\left\{p_{f} \mid f \in F\right\}^{\top}$, i.e., the rules use the maximally parallel application mode.

A computation in $\Pi$ starts from the initial configuration $\left(o_{E}, o_{1}, \ldots, o_{n}\right)$ where $o_{E} \in(O \backslash\{e\})^{*}$ and $o_{i}=e e, 1 \leq i \leq n$. The initial configuration of $k \Pi$ is $\left(\left[o_{E}, 0\right][e, 1][e, 1] \ldots[e, n][e, n], \lambda\right)$. In each cell $B_{i}, 1 \leq i \leq n$, at most one program, consisting of one or two rules, can be applied, and $B_{i}$ has always two objects inside. Those objects which are in the environment but are not affected by the activity of the cells are changed according to the rules in $P_{E}$. Similarly, in $k \Pi$ for any $i, 1 \leq i \leq n$, there are always two objects of the form $[a, i]$. Any configuration of $\Pi$ has the form $\left(x_{E}, x_{1}, \ldots, x_{n}\right)$, where $x_{E}$ is a multiset over $O \backslash\{e\}$ and $x_{i}, 1 \leq i \leq n$, is a multiset over $O$ with two objects. The corresponding configuration of $k \Pi$ is $\left(\left[x_{E}, 0\right]\left[x_{1}, 1\right] \ldots\left[x_{n}, n\right], y\right)$, where $\left[x_{i}, i\right], 1 \leq i \leq n$, denotes two objects $[a, i]$, as defined earlier in the proof. The object $\left[x_{E}, 0\right]$, as in the proof of Theorem 1 , corresponds to the $x_{E}$ from the environment of $\Pi$. It is easy to see that a program $p_{i, j}, 1 \leq i \leq n$, $1 \leq j \leq k_{i}$, is applied in $B_{i}$ of $\Pi$ if and only if the corresponding rule $p_{i, j}^{\prime}$ is applied in $C_{1}$ of $k \Pi$. Hence $x_{i}, 1 \leq i \leq n$, appears in a configuration of $\Pi$ if and only if $\left[x_{i}, i\right]$ appears in the corresponding configuration of $k \Pi$ and $x_{E}$ is in the environment of $\Pi$ if and only if $\left[x_{E}, 0\right]$ appears in $k \Pi$. The multiset $y$ is given by $y=x_{E} u u^{\prime}$, where $u=f_{1} \ldots f_{p}$ and $u^{\prime}=f_{1}^{\prime} \ldots f_{p}^{\prime}$. If no more rule simulating a program of $\Pi$ or corresponding to a rule of $D_{E}$ is applicable, then the multiset $u u^{\prime}$ is erased in the next step by applying rules of $R_{2}$ in a maximally parallel manner.

Thus, the two mechanisms have the same number of objects from $F$ in the environment ( $\mathrm{P}$ colony $\Pi)$ and in $C_{2}(\mathrm{kP}$ system $k \Pi)$ in a halting computation, hence $N(\Pi)=N(k \Pi)$.

\section{Producer/consumer example}

The producer/consumer paradigm consists of a system with two processors, a producer and a consumer, synchronising through a buffer of length 1 (accepting no more than an element). This has been modelled in different formalisms, including Petri nets [27] and generalised membrane systems using rewriting rules [1]. Recently, another approach based on generalised communicating $\mathrm{P}$ systems has been presented [17]. A slightly different version of this problem, using numerical $\mathrm{P}$ systems has been considered [24,25].

Here we model this problem using $\mathrm{P}$ colonies with senders and consumers and then consider it with $\mathrm{kP}$ systems. In this approach we use $\mathrm{P}$ colonies with senders and consumers having cells with both sender and consumer programs as they provide much simpler solutions to our problem.

The producer/consumer problem considered in this section can be described as follows: when the producer is informed that the buffer is empty, a signal that the buffer becomes full, followed by the current symbol are sent out to the consumer. After the symbol is consumed, the consumer responds back to the producer that the buffer is now empty. 
We consider a $\mathrm{P}$ colony with senders and consumers, $\Pi=\left(O, e, F, B_{1}, B_{2}\right)$, where the alphabet is $O=\{a, b, s, v, f\}$ and $B_{1}$ the producer cell and $B_{2}$ the consumer. Intially, $o_{1}=v f$ and $o_{2}=a b$. Please note that this is slightly different from the case studied before, whereby the initial multisets contain only multisets over $\{e\}$. It is easy to be observed that one can get the values for $o_{1}$ and $o_{2}$ with some simple initialisation rules starting from ee in both cells. We leave this as an exercise for the reader.

The set of final objects is not significant for this problem. The $\mathrm{P}$ colony with senders and consumers works as follows: the sender after receiving the signal that the buffer is empty (symbol $v$ - void, is received), sends to the environment a signal $f$ - full, that the buffer will become full and then the current symbol, $s$. These are sent to the environment and then retrieved by the consumer. We provide now the set of programs for the two cells.

$-P_{1}$, producer

$-p_{1}:<f_{\text {out }} ; v \rightarrow s f>$;

$-p_{2}:<s_{\text {out }} ; v \rightarrow$ af $>$;

$-p_{3}:<v_{i n} ;$ af $\rightarrow f>$;

- $P_{2}$, consumer

$-c_{1}:<f_{\text {in }} ; a b \rightarrow a>$;

$-c_{2}:<s_{i n} ; a f \rightarrow v>$;

$-c_{3}:<v_{\text {out }} ; s \rightarrow a b>$.

If $\left(x_{E}, x_{1}, x_{2}\right)$ and $\left(y_{E}, y_{1}, y_{2}\right)$ are two configurations and $p$ and $p^{\prime}$ the programs used to pass from the first to the second configuration then we write $\left(x_{E}, x_{1}, x_{2}\right) \Rightarrow^{p, p^{\prime}}\left(y_{E}, y_{1}, y_{2}\right)$. When one of the programs is not applicable then it is omitted.

One can write the following sequence of configurations starting from the initial one:

$$
\begin{gathered}
z_{1}=(\lambda, v f, a b) \Rightarrow^{p_{1}} z_{2}=(f, s f, a b) \Rightarrow^{p_{2}, c_{1}} z_{3}=(s, a f, a f) \Rightarrow^{c_{2}} \\
z_{4}=(\lambda, a f, s v) \Rightarrow^{c_{3}} z_{5}=(v, a f, a b) \Rightarrow^{p_{3}} z_{6}=(\lambda, v f, a b) .
\end{gathered}
$$

This shows that when the buffer is empty ( $v$ in $B_{1}$ in configuration $z_{1}$ ) then an $f$ is sent to the environment ( $f$ in configuration $z_{2}$ ) and then the symbol $s$ is sent to the environment (configuration $z_{3}$ ). These are then consumed by $B_{2}$. Symbol $v$ - corresponding to void buffer, is sent to the environmnet by $B_{2}$ (configuration $z_{5}$ ). In configuration $z_{6}$, the same as $z_{1}$, the void buffer is signalled to the producer, $B_{1}$.

Using the construction from the proof of Theorem 2 one can get a $\mathrm{kP}$ system that models the producer/consumer problem. This is left to the reader as a simple exercise. We prefer to provide a $\mathrm{kP}$ system with two compartments and equivalent to the one obtained from the $\mathrm{P}$ colony. The $\mathrm{kP}$ system is given by $k \Pi=\left(A, \mu, C_{1}, C_{2}\right)$. The output compartment is not significant and hence not considered above. The alphabet of objects is $A=\{a, b, c, v, f, s\}, \mu$ describes the connection between $C_{1}$ and $C_{2}$. The compartments are $C_{1}=\left(p, w_{1}\right)$, corresponding to producer, and $C_{2}=\left(c, w_{2}\right)$, corresponding to consumer, where 
$w_{1}=w_{2}=a$. The types $p$ and $c$ include the sets of rules $R_{1}$ and $R_{2}$, respectively. These are described below.

$$
\begin{aligned}
& -R_{1}, \text { producer } \\
& \quad-p_{1}^{\prime}: a \rightarrow b(f, c) ; \\
& \quad-p_{2}^{\prime}: b \rightarrow c(s, c) ; \\
& \quad-p_{3}^{\prime}: v c \rightarrow a ; \\
& -R_{2}, \text { consumer } \\
& \quad-c_{1}^{\prime}: f a \rightarrow b ; \\
& \quad-c_{2}^{\prime}: s b \rightarrow c ; \\
& \quad-c_{3}^{\prime}: c \rightarrow a(v, p) .
\end{aligned}
$$

The rules are applied with maximal parallelism in each compartment.

We use a notation similar to the one used for $\mathrm{P}$ colonies to define a sequence of configurations. We use two multisets of rules instead of programs. The following sequence can be obtained starting from the initial configuration:

$$
\begin{gathered}
z_{1}^{\prime}=(a, a) \Rightarrow^{\left\{p_{1}^{\prime}\right\}} z_{2}^{\prime}=(b, f a) \Rightarrow^{\left\{p_{2}^{\prime}\right\},\left\{c_{1}^{\prime}\right\}} z_{3}^{\prime}=(c, s b) \Rightarrow^{\left\{c_{2}^{\prime}\right\}} \\
z_{4}^{\prime}=(c, c) \Rightarrow^{\left\{c_{3}^{\prime}\right\}} z_{5}^{\prime}=(v c, a) \Rightarrow^{\left\{p_{3}^{\prime}\right\}} z_{6}^{\prime}=(a, a) .
\end{gathered}
$$

Although in the case of $\mathrm{kP}$ systems there is no environment, the components communicating directly, one can observe a very similar distribution of the key symbols - $v$ and $f$ related to the buffer status and $s$, generated by producer and received by consumer.

For the $\mathrm{kP}$ system model we are able to validate it by using the tools available within the kPWORKBENCH - a specification language for such models and model checkers [7]. We can simulate and also formally verify certain properties of the model. We present here some of the properties that we have considered for this model.

One can observe that the consumer (compartment $C_{2}$ ) consumes the current symbol, $s$, only after it is notified that the buffer is full, i.e., $f$ appears in $C_{2}$. This can be verified by considering the following property

$C_{2} . f>0$ followed-by $C_{2} . s>0$

and this can be expressed by the following specification of the model checker AG (C2.f $>0 \rightarrow E F C 2 . s>0)$,

which is true. The fact that the system has a cyclic behaviour, returning back to its initial state, containing $a$ in each of the two compartments, can be also formally proved by using

infinitely-often $C_{1} . a>0$.

This is expressed as

$A G(E F \quad C 1 . a>0)$,

which is also true. A similar property can be provided for $C_{2}$.a.

With such properties one can prove the correctness of our model specification, making sure the model describes accurately the problem. 


\section{Conclusions}

In this paper we have investigated the relationships among three classes of $\mathrm{P}$ colonies and kernel $\mathrm{P}$ systems. We have also showed how a synchronisation problem is represented by these formalisms. Some future research directions will include the possibility of enriching $\mathrm{P}$ colony models with features of the kernel $\mathrm{P}$ systems that might enhance the power and efficiency of the former. Another research avenue might be that of investigating the relationships with other P system models that will prove the flexibility of these classes of models in describing the behaviour of other computational models.

Acknowledgements The work of EC-V was supported by grant No. K 120558 of the NKFIH- National Research, Development, and Innovation Office, Hungary, MG and RL acknowledge the support provided by the Romanian National Authority for Scientific Research, CNCS-UEFISCDI (project number PN-III-P4- ID-PCE-2016-0210).

\section{References}

1. Bernardini, F., Gheorghe, M., Margenstern, M., Verlan, S.: Producer/consumer in membrane systems and Petri nets. In: 3rd Conference on Computability in Europe, CiE 2007, Lecture Notes in Computer Science, vol. 4495, pp. 43 - 52. Springer (2017)

2. Ciencialová, L., Cienciala, L., Sosík, P.: P colonies with evolving environment. In: Leporati et al. [19], pp. 151-164

3. Ciencialová, L., Csuhaj-Varjú, E., Cienciala, L., Sosík, P.: P colonies. Bulletin of the of the International Membrane Computing Society 2, 129-156 (2016)

4. Ciencialová, L., Csuhaj-Varjú, E., Kelemenová, A., Vaszil, G.: Variants of P colonies with very simple cell structure. International Journal of Computers, Communications and Control 4(3), $224-233$ (2009)

5. Csuhaj-Varjú, E., Kelemen, J., Kelemenová, A.: Computing with cells in environment: P colonies. Multiple-Valued Logic and Soft Computing 12(3-4), 201-215 (2006)

6. Dragomir, C., Ipate, F., Konur, S., Lefticaru, R., Mierla, L.: Model checking kernel P systems. In: A. Alhazov, S. Cojocaru, M. Gheorghe, Y. Rogozhin, G. Rozenberg, A. Salomaa (eds.) Membrane Computing - 14th International Conference, CMC 2013, Chişinău, Republic of Moldova, August 20-23, 2013, Revised Selected Papers, Lecture Notes in Computer Science, vol. 8340, pp. 151-172. Springer (2013)

7. Gheorghe, M., Ceterchi, R., Ipate, F., Konur, S.: Kernel P systems modelling, testing and verification - sorting case study. In: Leporati et al. [19], pp. 233-250

8. Gheorghe, M., Ipate, F., Dragomir, C.: Kernel P systems. Tenth Brainstorming Week on Membrane Computing pp. 153-170 (2012)

9. Gheorghe, M., Ipate, F., Dragomir, C., Mierla, L., Valencia-Cabrera, L., GarcíaQuismondo, M., Pérez-Jiménez, M.J.: Kernel P systems - version I. Eleventh Brainstorming Week on Membrane Computing (11BWMC) pp. 97-124 (2013)

10. Gheorghe, M., Ipate, F., Konur, S.: Kernel P systems and relationships with other classes of P systems. In: M. Gheorghe, I. Petre, M.J. Pérez-Jiménez, G. Rozenberg, A. Salomaa (eds.) Multidisciplinary Creativity, pp. 64-76. Spandugino Publishing House (2015)

11. Gheorghe, M., Ipate, F., Lefticaru, R., Pérez-Jiménez, M.J., Turcanu, A., ValenciaCabrera, L., García-Quismondo, M., Mierla, L.: 3-col problem modelling using simple kernel P systems. International Journal of Computer Mathematics 90(4), 816-830 (2013)

12. Gheorghe, M., Konur, S., Ipate, F.: Kernel P systems and stochastic P systems for modelling and formal verification of genetic logic gates. In: A. Adamatzky (ed.) Advances in Unconventional Computing: Volume 1: Theory, pp. 661-675. Springer International Publishing (2017) 
13. Gheorghe, M., Konur, S., Ipate, F., Mierla, L., Bakir, M.E., Stannett, M.: An integrated model checking toolset for kernel $\mathrm{P}$ systems. In: G. Rozenberg, A. Salomaa, J.M. Sempere, C. Zandron (eds.) Membrane Computing - 16th International Conference, CMC 2015, Valencia, Spain, August 17-21, 2015, Revised Selected Papers, Lecture Notes in Computer Science, vol. 9504, pp. 153-170. Springer (2015)

14. Kelemen, J., Kelemenová, A., Păun, Gh.: Preview of P colonies: A biochemically inspired computing model. In: Workshop and Tutorial Proceedings. Ninth International Conference on the Simulation and Synthesis of Living Systems (Alife IX), pp. 82-86. Boston, Massachusetts, USA (2004)

15. Kelemenová, A.: P colonies. In: Păun, Gh., G. Rozenberg, A. Salomaa (eds.) The Oxford Handbook of Membrane Computing, pp. 584-594. OUP Oxford (2010)

16. Krishna, S.N., Gheorghe, M., Dragomir, C.: Some classes of generalised communicating $\mathrm{P}$ systems and simple kernel P systems. In: P. Bonizzoni, V. Brattka, B. Löwe (eds.) The Nature of Computation. Logic, Algorithms, Applications - 9th Conference on Computability in Europe, CiE 2013, Milan, Italy, July 1-5, 2013. Proceedings, Lecture Notes in Computer Science, vol. 7921, pp. 284-293. Springer (2013)

17. Krishna, S.N., Gheorghe, M., Ipate, F., Csuhaj-Varjú, E., Ceterchi, R.: Further results on generalised communicating P systems. Theoretical Computer Science 701, 146 - 160 (2017)

18. Krithivasan, K., Păun, Gh., Ramanujan, A.: On controlled P systems. Fundamenta Informaticae 131(3 - 4), 451 - 464 (2014)

19. Leporati, A., Rozenberg, G., Salomaa, A., Zandron, C. (eds.): Membrane Computing - 17th International Conference, CMC 2016, Milan, Italy, July 25-29, 2016, Revised Selected Papers, Lecture Notes in Computer Science, vol. 10105. Springer (2017)

20. Mutyam, M., Krithivasan, K.: Generalized normal form for rewriting P systems. Acta Informatica 38(10), 721 - 734 (2002)

21. Mutyam, M., Prakash, V.J., Krithivasan, K.: Rewriting tissue P systems. Journal of Universal Computer Science 10(9), 1250 - 1271 (2004)

22. Păun, Gh.: Computing with membranes. Journal of Computer and System Sciences 61(1), 108-143 (2000)

23. Păun, Gh.: Membrane Computing - An Introduction. Springer (2002)

24. Păun, Gh., Păun, R.: Membrane computing as a framework for modeling economic processes. In: SYNASC05, Romania, IEEE Press, pp. 11 - 18 (2005)

25. Păun, Gh., Păun, R.: Membrane computing and economics. In: Păun, Gh., G. Rozenberg, A. Salomaa (eds.) The Oxford Handbook of Membrane Computing, pp. 632-644. OUP Oxford (2010)

26. Păun, Gh., Rozenberg, G., Salomaa, A. (eds.): The Oxford Handbook of Membrane Computing. Oxford University Press (2010)

27. Reisig, W.: Elements of distributed algorithms: modeling and analysis with Petri nets. Springer (1998) 